\section{Herzkranke leben länger mit mediterraner Ernährung}

\section{Mit einer mediterranen Kost könnten Herzkranke ihr Leben verlängern. Dar- auf deuten Ergebnisse einer großen Beobachtungsstudie hin.}

$S^{c}$ hon viel wurde über positive Effekte einer mediterranen Ernährung in der Primärprävention berichtet. Wenige Studien beschäftigten sich jedoch mit der Frage, ob eine Mittelmeerkost in der Sekundärprävention eine schützende Wirkung entfalten könnte. Anhaltspunkte dafür liefern die Ergebnisse der MONISANI-Studie, die von dem Studienleiter Dr. Giovanni de Gaetano aus Pozzilli beim ESC-Kongress vorgestellt wurden.

Bei knapp 1.200 kardiovaskulär erkrankten Personen aus der italienischen Region Molise war eine Ernährungsweise mit viel Fisch, Gemüse, Olivenöl und Co mit einer signifikant niedrigeren $\mathrm{Ge}$ samtmortalität assoziiert. So wiesen Teilnehmer, die sich sehr gewissenhaft mediterran ernährten, nach im Schnitt 7,3 Jahren eine um etwa $30 \%$ niedrigere Sterblichkeit auf als jene mit einer geringen Adhärenz zur mediterranen Kost.

\section{Gemüse besonders wichtig}

Um zu testen, welche Komponente der mediterranen Ernährung maßgeblich zum niedrigeren Risiko beiträgt, ließen die Wissenschaftler in einer statistischen Analyse je einen Nahrungsbestandteil weg. Demzufolge bietet vor allem Gemüse eine schützende Wirkung, die Effektgröße sank um $26 \%$ ohne diese Komponente, danach folgten Fisch (minus $23 \%$ ), Früchte und Nüsse (minus 13,4\%) und ein Verhältnis zugunsten von mehrfach ungesättigten Fettsäuren (minus 12,9\%).

Gaetano zufolge deuten diese Daten an, dass eine mediterrane Kost in der Sekundärprävention präventiv wirken könnte. „Ärzte können Medikamente nicht als einzige Option ansehen, um weitere kardiovaskuläre Ereignisse zu verhindern“, betonte er.

\section{Gutes Essen ist teuer}

Auffällig ist, dass nach Adjustierung auf den sozioökonomischen Status der beobachtete Effekt schwindet. Gaetano erklärt sich dies mit der ökonomischen Herausforderung für Geringverdiener, sich mediterran zu ernähren. Wie soll sich eine vierköpfige Familie zweimal pro Woche
Fisch und fünf Portionen Obst und Gemüse täglich leisten können. Die Politik sollte seiner Meinung darauf hinwirken, dass nicht nur die Kosten für Medikamente übernommen werden, sondern auch eine gesunde Lebensweise der Patienten finanziell unterstützt wird.

Jedoch wird aus der Studie nicht ersichtlich, wie die Mortalität durch die Mittelmehrkost gesenkt wird: Sind es tatsächlich Effekte auf Herz-Kreislauf oder Krebserkrankungen oder liegt es an etwas ganz anderem, etwa an der gesünderen Lebensweise von sich mediterran ernährenden Menschen? Auch wenn in die-

ser Analyse auf den BMI, körperliche Aktivität, Raucherstatus usw. adjustiert wurde, lassen sich nicht alle Einflussfaktoren komplett ausschließen.

Die Studie habe nur eine statistisch signifikante Assoziation gezeigt, so Gaetano. Das müsse nicht bedeuten, dass ein kausaler Zusammenhang zwischen einer Mittelmeerkost und der Mortalität bestehe.

Veronika Schlimpert

Pressekonferenz „Preventing Sudden Death Diet or Device", 29.08.16, ESC-Kongress in Rom

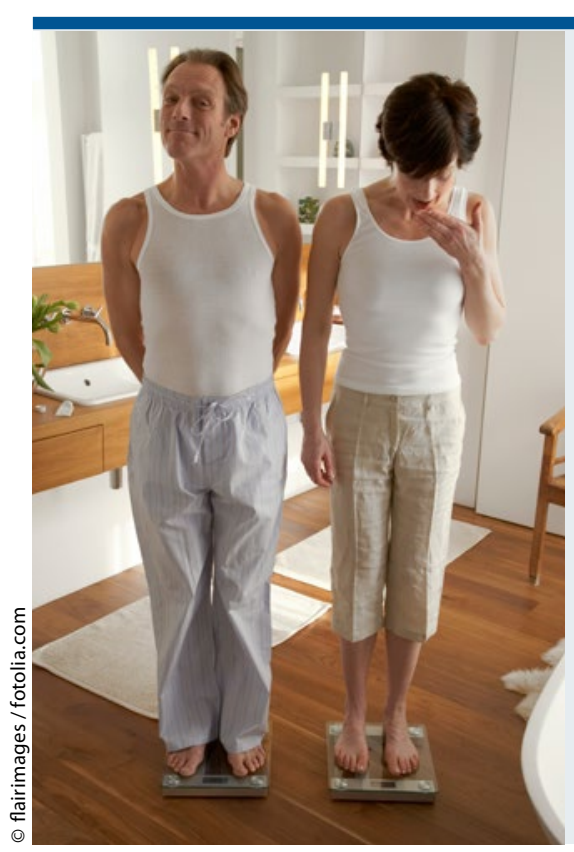

Glosse

\title{
Zufriedenheit geht über alles!
}

Fast zwei Drittel aller Deutschen sind mit ihrem Gewicht und ihrer Fitness durchaus zufrieden. In den letzten Jahren sind es sogar mehr geworden, so das Ergebnis einer kürzlich publizierten Umfrage. Auch wenn dieses Maß an Selbstzufriedenheit uns Ärzte etwas überrascht, ja wenn wir darin sogar eine wahnhaft anmutende Verkennung der Realität sehen mögen, drängt sich doch die Frage auf, warum wir uns immer wieder als Spielverderber oder Spaßbremse profilieren müssen.

"Sie müssen abnehmen und mehr Sport treiben“, dieses Präventions-Mantra können wir bald selbst nicht mehr hören und bringen tut es auch wenig. Und ad usum proprium sind solche Ermahnungen meist nicht gedacht, wie ein Blick ins Auditorium bei Ärztefortbildungen zeigt. Aber Wegweiser sollen bekanntlich ja nicht mitgehen. Sollten wir nicht lieber über "kleine" Dysmorphien großzügig hinwegschauen und unserem Patienten häufiger zu seinem „Körper" gratulieren. Sollten wir ihm nicht lieber dazu raten, Spiegel und Waagen aus der Wohnung zu verbannen - soweit er es nicht schon getan hat - bevor Selbstzweifel aufkommen. Selbstzufriedenheit ist eine wichtige Tugend und wir Ärzte sollten unseren Patienten dabei helfen, sie zu erlangen; auch wenn die Diskrepanz zwischen objektiver und subjektiver Wahrnehmung zumindest gelegentlich unübersehbar ist. Und glückliche Patienten leben länger, wohingegen es jenen, für die die Prävention zur Lebensaufgabe geworden ist, oft nur so vorkommt.

Dr. med. Peter Stiefelhagen 